(2) OPEN ACCESS
- Additional material is published online only. To view please visit the journal online (http://dx.doi.org/10.1136/ oemed-2019-106013).

Correspondence to Tim Driscoll, School of Public Health, University of Sydney, Sydney, NSW 2006, Australia; tim.driscoll@sydney.edu.au

Received 5 June 2019 Revised 13 October 2019 Accepted 30 November 2019

\section{Linked}

- http://dx.doi.org/10.1136/ oemed-2019-106349

Check for updates

(C) Author(s) (or their employer(s)) 2020. Re-use permitted under CC BY. Published by BMJ.

To cite: GBD 2016

Occupational Chronic Respiratory Risk

Factors Collaborators.

Occup Environ Med

2020:77:142-150.

\title{
Global and regional burden of chronic respiratory disease in 2016 arising from non-infectious airborne occupational exposures: a systematic analysis for the Global Burden of Disease Study 2016
}

\author{
GBD 2016 Occupational Chronic Respiratory Risk Factors Collaborators
}

\section{ABSTRACT}

Objectives This paper presents detailed analysis of the global and regional burden of chronic respiratory disease arising from occupational airborne exposures, as estimated in the Global Burden of Disease 2016 study. Methods The burden of chronic obstructive pulmonary disease (COPD) due to occupational exposure to particulate matter, gases and fumes, and secondhand smoke, and the burden of asthma resulting from occupational exposure to asthmagens, was estimated using the population attributable fraction (PAF), calculated using exposure prevalence and relative risks from the literature. PAFs were applied to the number of deaths and disability-adjusted life years (DALYs) for COPD and asthma. Pneumoconioses were estimated directly from cause of death data. Age-standardised rates were based only on persons aged 15 years and above. Results The estimated PAFs (based on DALYs) were 17\% (95\% uncertainty interval (UI) 14\%-20\%) for COPD and 10\% (95\% UI 9\%-11\%) for asthma. There were estimated to be 519000 (95\% UI 441,000$609,000)$ deaths from chronic respiratory disease in 2016 due to occupational airborne risk factors (COPD: 460, 100 [95\% UI 382,000-551,000]; asthma: 37,600 [95\% UI 28,400-47,900]; pneumoconioses: 21,500 $[95 \%$ UI 17,900-25,400]. The equivalent overall burden estimate was 13.6 million (95\% UI 11.9-15.5 million); DALYS (COPD: 10.7 [95\% UI 9.0-12.5] million; asthma: 2.3 [95\% UI 1.9-2.9] million; pneumoconioses: 0.58 [95\% UI 0.46-0.67] million). Rates were highest in males; older persons and mainly in Oceania, Asia and sub-Saharan Africa; and decreased from 1990 to 2016. Conclusions Workplace exposures resulting in COPD, asthma and pneumoconiosis continue to be important contributors to the burden of disease in all regions of the world. This should be reducible through improved prevention and control of relevant exposures.

\section{INTRODUCTION}

Airborne respiratory hazards (inorganic and organic particulate matter, vapours, gases and fumes) are a common exposure in occupational settings and many studies have identified resulting malignant and chronic respiratory disease as an important component of the occupational injury and disease burden at both country and global levels. ${ }^{1-10}$ Workrelated respiratory diseases remain a problem even

\section{Key messages}

What is already known about this subject?

- Occupational respiratory exposures have been shown to be an important cause of chronic work-related respiratory disease at national and global level.

- The last analysis of this issue at the global level was for the year 2000 - this paper provides a new analysis for 2016.

What are the new findings?

- Analysis of the Global Burden of Disease data set suggests that globally there were about 519,100 deaths and 13.6 million disabilityadjusted life years in 2016 from chronic respiratory disease due to occupational airborne exposures.

- The population attributable fraction for chronic obstructive pulmonary disease (COPD) was 17\% and for asthma was $10 \%$.

- Workplace exposures resulting in COPD, asthma and pneumoconioses remain important contributors to the burden of disease in all regions of the world.

How might this impact on policy or clinical practice in the foreseeable future?

- These findings highlight opportunities to continue to reduce chronic respiratory disease burden worldwide by improving prevention and control of workplace airborne exposures.

in high-income countries, as shown by incident cases of pneumoconioses that are still occurring. ${ }^{1112}$

The Global Burden of Disease (GBD) Comparative Risk Assessment (CRA) project was the first to consider the burden of occupational chronic respiratory disease comprehensively at a regional and global level, estimating the burden for the year $2000{ }^{13}$ That study included airborne exposures leading to asthma, chronic obstructive pulmonary disease (COPD), asbestosis, coal workers' pneumoconiosis (CWP) and silicosis. ${ }^{14}$

The new GBD initiative, conducted by the Institute of Health Metrics and Evaluation, first focused on $2010^{15}$ and has been updated several times since. ${ }^{16-19}$ It provides a detailed analysis of the 
burden of disease and injury overall and arising from specific risk factors. One set of those risk factors comprises occupational exposures, ${ }^{18}$ but no detailed analysis of the occupational risk factor results has been undertaken.

This paper presents a detailed analysis of the global and regional burden of chronic respiratory disease arising from noninfectious occupational airborne exposures, as estimated in the GBD 2016 study. Malignant occupational respiratory disease ${ }^{20}$ and an overview of all occupational risk factors ${ }^{21}$ are considered in companion papers. ${ }^{20}$

\section{METHODS}

\section{General approach}

The general methodology used in GBD 2016 is described elsewhere, ${ }^{18}$ as is the overall approach to occupational risk factors. ${ }^{21}$ These methods are briefly summarised here. A more detailed description is provided here of the analyses of occupational exposures to particulate matter, gases and fumes (PMGF), secondhand smoke (SHS), asthmagens and pneumoconiotic dusts and their associated outcomes.

The burden of occupational respiratory disease for PMGF and SHS (causing COPD) and for asthmagens was estimated using the population attributable fraction (PAF), that is, the proportion of deaths or disability-adjusted life years (DALYs) that would not have occurred if exposure was at the theoretical minimum risk exposure level; this was then used to estimate attributable numbers of deaths or DALYs. The PAF requires information on the relative risk of the disease due to the exposure of interest and the proportion of the target population exposed. Pneumoconioses were estimated directly as part of the overall GBD estimates of prevalence and deaths for each included cause. Age-standardised rates (per 100000 people) were based only on persons aged 15 and above. Results were calculated for all years from 1990 to 2016, inclusive; the 2016 findings are the focus of this paper. The socio-demographic index (SDI) is a composite indicator of development status based on total fertility rate, mean education for those aged 15 and older and lag distributed income per capita. ${ }^{18}$ Region-specific, SDI-specific and global results are reported here. Country-specific information is available through the GBD Compare data visualisation. ${ }^{22} \mathrm{High}-$ income countries were defined as countries in the Australasia, high-income North America, Western Europe and Asia Pacific regions, and low/middle-income (LMI) countries as all other countries. Employment data came from the International Labour Organization Labour Force, ${ }^{23}$ supplemented where necessary by sub-national data sources and modelling. PAFs for all carcinogens except asbestos were estimated for each age-sex-country group using the equation based on $\operatorname{Levin}^{24}$ :

$$
\mathrm{PAF}=\frac{\sum_{\mathrm{x}=1}^{\mathrm{n}} \mathrm{RR}(\mathrm{x}) \mathrm{P}(\mathrm{x})-1}{\sum_{\mathrm{x}=1}^{\mathrm{n}} \mathrm{RR}(\mathrm{x}) \mathrm{P}(\mathrm{x})}
$$

where $\mathrm{P}(\mathrm{x})$ is the proportion of persons exposed at level $\mathrm{x}$ in the relevant population and $\mathrm{RR}(\mathrm{x})$ is the relative risk corresponding to exposure level $\mathrm{x}$.

\section{PMGF and SHS}

Industry was used as a proxy for exposure to PMGF because we identified no suitable and valid data sources at a country or global level of exposure to PMGF, either singly or to PMGF as a group. Current industry was used as the basis of exposure estimates, but the estimates of proportions exposed (ie, workers who experienced more than trivial exposure) within each industry (nine categories-see online supplementary table S1) were designed to take into account past exposure (to estimate ever exposed), given that both past and current exposure appear to increase the risk of COPD. Estimates of proportion exposed at lower and higher levels in high income and LMI countries were based on sparse published data (see online supplementary material) and expert opinion by GBD collaborators (online supplementary table S1). Information on risk was obtained by conducting a systematic review of international literature and meta-analysis (unpublished) of relevant results. ${ }^{525}$ Relative risks in these studies were for COPD greater than or equal toGlobal Initiative for Chronic Obstructive Lung Disease(GOLD) stage II: defined as requiring non-reversibility after using bronchodilators for provocation, a forced expiratory volume in one second/forced vital capacity $\left(\mathrm{FEV}_{1} /\right.$ FVC) ratio of less than 0.70 and an $\mathrm{FEV}_{1}$ of less than $80 \%$ predicted. ${ }^{26}$ Relative risk estimates were used for an overall 'lower' level ( $\mathrm{RR}=1.44$; 95\% CI 1.07-1.95) and an overall 'higher' level ( $\mathrm{RR}=2.31$; $95 \% \mathrm{CI} 1.45$ to 3.73 ) of exposure to the agents of concern ('higher' and 'lower' were based on the exposure descriptions in the papers). ${ }^{525}$ The reference group was persons not working and persons working in trade, finance or service industries. The prevalence of exposure to PMGF was determined using the following equation:

$$
\text { 1) Prevalence of Exposure } e_{c, y, s, a, l}=\sum_{\mathrm{EA}} \text { Proportion }_{\mathrm{EAC}, y} * \mathrm{EAP}_{c, y, s,}
$$$$
\text { *Exposure level proportion }{ }_{\mathrm{EAl}}
$$

where $\mathrm{EAP}=$ economically active population, $\mathrm{c}=$ country, $\mathrm{s}$ $=$ sex, $\mathrm{EA}=$ economic activity, $\mathrm{l}=$ level of exposure, $\mathrm{y}=\mathrm{year}$ and $\mathrm{a}=$ age.

Exposure information on SHS was based on the CAREX (Carcinogen Exposure) database, which provides industryspecific information from 1990 to 1993 on the prevalence of exposure to various carcinogens in countries of Western Europe, ${ }^{27}$ as described elsewhere. ${ }^{20}$ The relevant relative risks were those used for SHS in the general GBD 2016 analysis. ${ }^{18}$

\section{Asthmagens}

Exposure and relative risks for asthmagens were based on the current occupation distribution (eight categories-see online supplementary table S2) because there were no suitable and valid data sources at a country or global level describing exposure to the wide range of occupational asthmagens. All relative risk information, except that for agricultural occupations, came from a study by Karjalainen and coworkers, a comprehensive national population study of incident asthma. ${ }^{78}$ Relative risks for agricultural occupations were based on a study by Kogevinas and coworkers ${ }^{28}$ using a weighted average of the separate estimates for 'farmers' and 'agricultural' workers provided in the paper. This information was used because the results were thought to be more generalisable to agriculture in the rest of the world, especially for LMI regions. Separate risks were available and used for males and females (except for agricultural operations), although the sex-specific risks were similar and within the limits of random variation. The same relative risks were used for all age groups. The counterfactual was persons not working and administrative workers. Byssinosis was included as asthma for the purposes of the analysis. The prevalence of exposure to asthmagens was determined using the following equation:

$$
\text { 2) Prevalence of Exposure } e_{c, y, s, a}=\sum_{\mathrm{EA}} \text { Proportion }_{\mathrm{occc}, y} * \mathrm{EAP}_{c, y, \mathrm{~s},}
$$

where $\mathrm{EAP}=$ economically active population, $\mathrm{c}=$ country, $\mathrm{s}$ $=$ sex, OCC $=$ occupation, $\mathrm{y}=$ year and $\mathrm{a}=$ age. 


\section{Pneumoconiotic dusts}

As mentioned, pneumoconioses were estimated directly as part of the overall GBD estimates of prevalence and deaths for each included cause, rather than using the attributable fraction approach. The methods used are described elsewhere. ${ }^{29}$ The attributable fraction is essentially $100 \%$ because virtually all pneumoconioses arise as a result of occupational exposure. Separate estimates were available for silicosis, asbestosis and CWP, with the remaining cases grouped under an 'other pneumoconiosis' category. ${ }^{23}$

\section{Statistical approach}

The main modelling and analyses employed to produce the GBD 2016 data, and the calculation and use of $95 \%$ uncertainty intervals (95\% UI), were as described elsewhere. ${ }^{1821}$ Uncertainty intervals are primarily presented in detail in the tables to assist with the flow of the text.

\section{RESULTS}

There were estimated to be about $519000 \quad(95 \%$ UI 441000-609000) deaths from chronic respiratory disease in 2016 due to occupational airborne risk factors. The vast majority (460000 [95\% UI 382000-551000]; 89\%) of these were due to COPD arising from PMGF and SHS. The remaining deaths were from asthma (37 600 [95\% UI 28 400-47 900]; 7\%), due to exposure to a range of asthmagens, and from pneumoconiosis (21500 [95\% UI 17900-25 400]; 4\%), arising from exposure to pneumoconiotic dusts. Males accounted for $75 \%$ (390000) of the deaths overall and between 69\% (asthmagens) and $88 \%$ (pneumoconiotic dusts) for individual risk factors. The relative contribution of the different risk factors was similar when the burden was measured in terms of DALYs (13.6 [95\% UI 11.9-15.5] million DALYs overall; COPD: 10.7 [95\% UI 9.0-12.5] million; asthma: 2.3 [95\% UI 1.9-2.9] million; pneumoconioses: 0.58 [95\% UI 0.49-0.67] million), with $79 \%$ of the DALYs due to PMGF and SHS. Males accounted for 73\% (9.9 million) of the DALYs (table 1).

\section{PMGF and SHS}

The PAF for COPD arising from occupational exposures was $17 \%$ (95\% UI 14\%-20\%) for DALYs (16\% for deaths), ranging from a low of $10 \%$ in Central sub-Saharan Africa to $21 \%$ in East Asia (table 2). The PAF was much higher in males (21\%) than females (11\%) and peaked at about $24 \%$ in 60-64-year-old males. The highest number of deaths and the highest rate of deaths from COPD due to occupational exposures occurred in the older age groups, often beyond usual retirement age. Males had three to four times the number and rate of deaths compared with females. The peak for number of DALYs occurred in the 65-74 year age group, but the rate of DALYs increased considerably with age and was highest in the 75-84 year group for both males and females (online supplementary figures S1-S4).

By far the highest number of deaths and DALYs from COPD occurred in East Asia and South Asia, the regions with the largest populations, which together accounted for about $71 \%$ of both measures. The highest rates of deaths were in Oceania, South Asia and East Asia, the rates in these three regions being considerably higher than elsewhere (the lowest rates were in highincome Asia Pacific and Eastern Europe).

The same regions had the highest DALY rates (the lowest DALY rates were in Andean Latin America, high-income Asia Pacific and the Caribbean). Rates tended to be higher in low-middle and middle SDI regions, and there was considerable variation between regions.

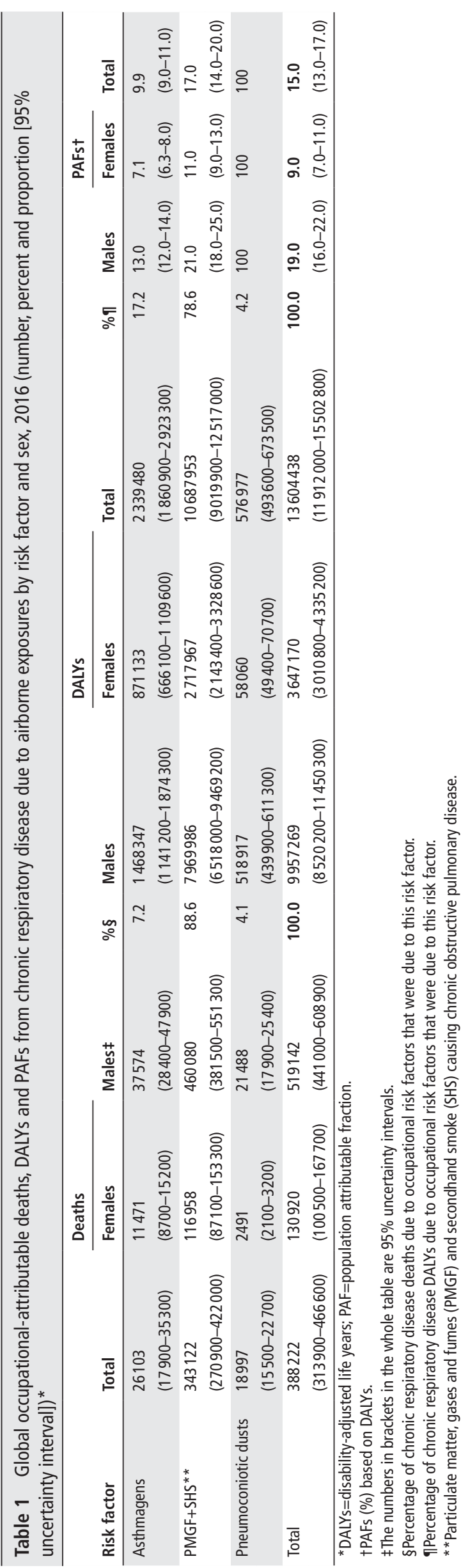


Table 2 Deaths, YLLs, YLDs, DALYs and PAFs from COPD due to occupational exposure to PMGF and SHS, by region, 2016 (number, percent, rate, proportion)*

\begin{tabular}{|c|c|c|c|c|c|c|c|c|c|c|c|c|c|c|}
\hline & Deaths & Deaths & YLLs & YLDs & DALYs & DALYs & Deaths & YLLs & YLDs & DALYs & YLL & YLD & PAF & PAF \\
\hline Region & number & $\%$ & number & number & number & $\%$ & ratet & ratet & ratet & ratet & $\% \ddagger$ & $\% \ddagger$ & (deaths)§ & (DALYs)ף \\
\hline High-income North America & 16669 & 3.6 & 287084 & 154716 & 441800 & 4.1 & 9.8 & 173 & 95 & 267 & 65.0 & 35.0 & 9.4 & 11.9 \\
\hline Australasia & 1047 & 0.2 & 15975 & 4922 & 20897 & 0.2 & 7.7 & 122 & 39 & 161 & 76.4 & 23.6 & 10.0 & 13.1 \\
\hline High-income Asia Pacific & 5150 & 1.1 & 61981 & 70847 & 132828 & 1.2 & 4.8 & 61 & 76 & 136 & 46.7 & 53.3 & 12.0 & 15.5 \\
\hline Western Europe & 18258 & 4.0 & 250656 & 73485 & 324141 & 3.0 & 7.8 & 113 & 35 & 148 & 77.3 & 22.7 & 9.1 & 11.6 \\
\hline Southern Latin America & 2844 & 0.6 & 44526 & 4967 & 49493 & 0.5 & 10.8 & 174 & 20 & 194 & 90.0 & 10.0 & 12.2 & 14.3 \\
\hline Eastern Europe & 6391 & 1.4 & 119566 & 21018 & 140584 & 1.3 & 6.3 & 119 & 21 & 140 & 85.0 & 15.0 & 12.7 & 13.6 \\
\hline Central Europe & 4622 & 1.0 & 78183 & 20299 & 98482 & 0.9 & 7.9 & 136 & 36 & 172 & 79.4 & 20.6 & 12.2 & 13.9 \\
\hline Central Asia & 2584 & 0.6 & 51612 & 8277 & 59889 & 0.6 & 10.1 & 190 & 29 & 219 & 86.2 & 13.8 & 16.4 & 16.8 \\
\hline Central Latin America & 7042 & 1.5 & 107850 & 25554 & 133404 & 1.2 & 9.3 & 139 & 31 & 171 & 80.8 & 19.2 & 13.6 & 15.1 \\
\hline Andean Latin America & 1241 & 0.3 & 17903 & 4217 & 22120 & 0.2 & 7.1 & 99 & 23 & 122 & 80.9 & 19.1 & 15.3 & 16.1 \\
\hline Caribbean & 1191 & 0.3 & 20636 & 3576 & 24213 & 0.2 & 7.2 & 126 & 22 & 147 & 85.2 & 14.8 & 13.2 & 14.2 \\
\hline Tropical Latin America & 9936 & 2.2 & 179699 & 25150 & 204849 & 1.9 & 13.7 & 239 & 32 & 271 & 87.7 & 12.3 & 15.1 & 17.3 \\
\hline East Asia & 171300 & 37.2 & 2634163 & 592605 & 3226768 & 30.2 & 31.5 & 461 & 99 & 560 & 81.6 & 18.4 & 18.8 & 20.5 \\
\hline Southeast Asia & 28029 & 6.1 & 508878 & 350306 & 859183 & 8.0 & 15.0 & 252 & 164 & 415 & 59.2 & 40.8 & 18.3 & 18.7 \\
\hline Oceania & 886 & 0.2 & 21518 & 3182 & 24700 & 0.2 & 35.9 & 767 & 107 & 874 & 87.1 & 12.9 & 13.9 & 13.1 \\
\hline North Africa and Middle East & 9834 & 2.1 & 193777 & 69764 & 263541 & 2.5 & 6.9 & 125 & 42 & 167 & 73.5 & 26.5 & 12.4 & 12.8 \\
\hline South Asia & 157117 & 34.1 & 3049410 & 1176092 & 4225502 & 39.5 & 34.8 & 626 & 227 & 853 & 72.2 & 27.8 & 15.9 & 16.5 \\
\hline Southern sub-Saharan Africa & 2117 & 0.5 & 39719 & 15450 & 55169 & 0.5 & 11.2 & 197 & 72 & 269 & 72.0 & 28.0 & 12.7 & 12.3 \\
\hline Western sub-Saharan Africa & 4775 & 1.0 & 105149 & 39099 & 144248 & 1.3 & 7.9 & 144 & 49 & 192 & 72.9 & 27.1 & 16.2 & 15.6 \\
\hline Eastern sub-Saharan Africa & 7716 & 1.7 & 154524 & 46238 & 200761 & 1.9 & 12.3 & 217 & 60 & 277 & 77.0 & 23.0 & 19.4 & 18.3 \\
\hline Central sub-Saharan Africa & 1333 & 0.3 & 27051 & 8328 & 35379 & 0.3 & 7.3 & 130 & 36 & 166 & 76.5 & 23.5 & 9.8 & 9.5 \\
\hline High SDI & 41455 & 9.0 & 630080 & 319949 & 950029 & 8.9 & 7.7 & 122 & 65 & 187 & 66.3 & 33.7 & 9.8 & 12.4 \\
\hline High-middle SDI & 55985 & 12.2 & 855050 & 280400 & 1135450 & 10.6 & 12.6 & 191 & 63 & 253 & 75.3 & 24.7 & 15.7 & 17.4 \\
\hline Middle SDI & 185785 & 40.4 & 3067814 & 1016253 & 4084066 & 38.2 & 24.0 & 377 & 110 & 486 & 75.1 & 24.9 & 17.9 & 19.3 \\
\hline Low-middle SDI & 156339 & 34.0 & 2995849 & 971878 & 3967727 & 37.1 & 29.5 & 522 & 169 & 691 & 75.5 & 24.5 & 15.9 & 16.2 \\
\hline Low SDI & 20517 & 4.5 & 421068 & 129612 & 550681 & 5.2 & 16.2 & 291 & 83 & 374 & 76.5 & 23.5 & 15.2 & 14.9 \\
\hline Global & 460080 & 100.0 & 7969861 & 2718092 & 10687953 & 100.0 & 18.1 & 309 & 103 & 412 & 74.6 & 25.4 & 15.7 & 16.8 \\
\hline
\end{tabular}

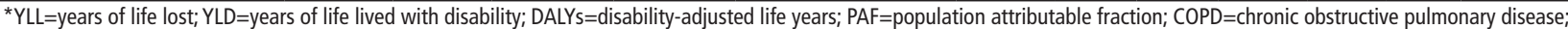

PMGF=particulate matter, gases and fumes; $\mathrm{SHS}=$ secondhand smoke.

tPer 100000 persons.

‡Percentage of DALYs.

§Percentage of all COPD deaths due to occupational exposures.

१Percentage of all COPD DALYs due to occupational exposures.

SDI, socio-demographic index.

Seventy-five percent of the DALYs were due to years of life lost (YLLs); this predominance of YLLs was seen in nearly all regions (table 2). Information on the separate contribution of SHS to COPD is presented in the online supplementary material.

\section{Asthmagens}

The PAF for asthma from occupational exposures was estimated to be $9.9 \%$ (95\% UI 9.0\%-10.9\%) based on DALYs (8.9\% [95\% UI 7.8-10.1\%] based on deaths), ranging from $4.1 \%$ in Central sub-Saharan Africa to $12.0 \%$ in South Asia (table 3). The PAF was higher for males (13\%) than females (7\%) and peaked at around $18 \%$ between the ages of about 35 and 49 years.

Deaths arising from occupational exposure to asthmagens occurred at all ages from 15 to 79 years, but the highest numbers occurred in persons aged 55-64 and the highest rates in persons aged 65-79. The burden was spread more evenly across age groups in terms of DALYs, with the highest number of DALYs in the 45-54 year age group and the highest rates in the 55-64 year age group (online supplementary figures S5-S8).

The highest number of deaths occurred in South Asia and Southeast Asia, and rates were highest in the low and low-middle SDI regions, particularly Oceania, South Asia and Southeast Asia (the lowest rates were in Western Europe and Central Europe). A similar pattern was seen for DALYs (the lowest rates were in
East Asia and Tropical Latin America). Overall, YLLs and years of life with disability each contributed about $50 \%$ to the DALYs. However, low and low-middle SDI regions had a much higher proportion of DALYs due to YLLs compared with high-income regions, reflecting that a higher proportion of deaths occurred at younger ages in these regions compared with the high and highmiddle SDI regions (table 3).

\section{PNEUMOCONIOTIC DUSTS}

The PAF for all pneumoconioses was assumed to be $100 \%$. Silicosis (48\%) was the largest specific cause of death from pneumoconiosis, ahead of asbestosis (16\%) and CWP (12\%), but about one-quarter of the deaths were classified in the 'other pneumoconiosis' category. There was a similar distribution between pneumoconiosis categories in terms of DALYs (table 4). The number of deaths increased with age until age 85 years and over, and the age-standardised death rates were highest in the older age groups. There was a broader distribution of DALYs across age groups, and although the rates still increased with increasing age, the rate was highest in the 75-84 year age group (online supplementary figures S9-S12).

The highest number of deaths and DALYs overall and for silicosis and CWP occurred in East Asia, South Asia and Western Europe, with high-income North America replacing East Asia for 
Table 3 Deaths, YLLs, YLDs, DALYs and PAFs from asthma due to occupational exposure to asthmagens, by region, 2016 (number, percent, rate, proportion)*

\begin{tabular}{|c|c|c|c|c|c|c|c|c|c|c|c|c|c|c|}
\hline & Deaths & Deaths & YLLs & YLDs & DALYs & DALYs & Deaths & YLLs & YLDs & DALYs & YLL & YLD & PAF & PAF \\
\hline Region & number & $\%$ & number & number & number & $\%$ & ratet & ratet & ratet & ratet & $\% \ddagger$ & $\% \ddagger$ & (deaths)§ & (DALYs)ף \\
\hline High-income North America & 388 & 1.0 & 15269 & 71605 & 86873 & 3.7 & 0.3 & 10.6 & 50.2 & 60.7 & 17.6 & 82.4 & 8.8 & 10.7 \\
\hline Australasia & 23 & 0.1 & 857 & 11291 & 12148 & 0.5 & 0.2 & 7.4 & 100.1 & 107.5 & 7.1 & 92.9 & 4.3 & 9.0 \\
\hline High-income Asia Pacific & 135 & 0.4 & 3493 & 34431 & 37923 & 1.6 & 0.2 & 4.2 & 46.3 & 50.5 & 9.2 & 90.8 & 2.6 & 8.0 \\
\hline Western Europe & 182 & 0.5 & 6525 & 94309 & 100834 & 4.3 & 0.1 & 3.6 & 55.8 & 59.4 & 6.5 & 93.5 & 2.8 & 8.4 \\
\hline Southern Latin America & 46 & 0.1 & 1558 & 12849 & 14407 & 0.6 & 0.2 & 6.2 & 51.6 & 57.8 & 10.8 & 89.2 & 4.9 & 7.5 \\
\hline Central Asia & 166 & 0.4 & 5423 & 13953 & 19376 & 0.8 & 0.6 & 17.5 & 42.9 & 60.4 & 28.0 & 72.0 & 8.0 & 9.4 \\
\hline Central Latin America & 185 & 0.5 & 6315 & 32529 & 38844 & 1.7 & 0.2 & 7.0 & 34.3 & 41.4 & 16.3 & 83.7 & 5.7 & 6.8 \\
\hline Andean Latin America & 31 & 0.1 & 952 & 8977 & 9929 & 0.4 & 0.2 & 4.8 & 42.0 & 46.7 & 9.6 & 90.4 & 7.4 & 6.8 \\
\hline Caribbean & 140 & 0.4 & 5049 & 8442 & 13491 & 0.6 & 0.8 & 29.6 & 49.3 & 78.9 & 37.4 & 62.6 & 7.5 & 6.5 \\
\hline Tropical Latin America & 176 & 0.5 & 6291 & 22647 & 28937 & 1.2 & 0.2 & 7.5 & 26.7 & 34.2 & 21.7 & 78.3 & 6.3 & 7.2 \\
\hline South Asia & 21085 & 56.1 & 669102 & 223370 & 892472 & 38.1 & 3.9 & 117.5 & 37.9 & 155.4 & 75.0 & 25.0 & 9.7 & 12.0 \\
\hline Southern sub-Saharan Africa & 474 & 1.3 & 17146 & 13274 & 30420 & 1.3 & 2.0 & 69.4 & 49.5 & 118.9 & 56.4 & 43.6 & 7.7 & 8.0 \\
\hline Western sub-Saharan Africa & 1395 & 3.7 & 50249 & 51856 & 102105 & 4.4 & 1.5 & 50.7 & 45.3 & 96.1 & 49.2 & 50.8 & 10.7 & 8.5 \\
\hline Eastern sub-Saharan Africa & 2452 & 6.5 & 85585 & 75354 & 160939 & 6.9 & 2.9 & 91.8 & 68.2 & 160.0 & 53.2 & 46.8 & 13.2 & 10.7 \\
\hline Central sub-Saharan Africa & 316 & 0.8 & 11315 & 10173 & 21487 & 0.9 & 1.2 & 41.1 & 33.1 & 74.1 & 52.7 & 47.3 & 5.1 & 4.1 \\
\hline High SDI & 776 & 2.1 & 27685 & 213996 & 241681 & 10.3 & 0.2 & 6.5 & 52.1 & 58.6 & 11.5 & 88.5 & 4.6 & 9.2 \\
\hline High-middle SDI & 1320 & 3.5 & 44449 & 185336 & 229785 & 9.8 & 0.3 & 9.1 & 38.1 & 47.2 & 19.3 & 80.7 & 6.2 & 9.1 \\
\hline Middle SDI & 8807 & 23.4 & 277589 & 316569 & 594158 & 25.4 & 1.0 & 30.5 & 34.5 & 65.0 & 46.7 & 53.3 & 8.1 & 9.3 \\
\hline Low-middle SDI & 21524 & 57.3 & 683953 & 297559 & 981512 & 42.0 & 3.3 & 101.3 & 41.4 & 142.7 & 69.7 & 30.3 & 9.7 & 11.1 \\
\hline Low SDI & 5147 & 13.7 & 178483 & 113860 & 292343 & 12.5 & 2.9 & 94.3 & 52.3 & 146.5 & 61.1 & 38.9 & 9.9 & 8.9 \\
\hline Global & 37574 & 100.0 & 1212160 & 1127320 & 2339480 & 100.0 & 1.4 & 44.0 & 40.5 & 84.5 & 51.8 & 48.2 & 8.9 & 9.9 \\
\hline
\end{tabular}

${ }^{*}$ YLL=years of life lost; YLD=years of life lived with disability; DALYs=disability-adjusted life years; PAF=population attributable fraction.

†Per 100000 persons.

‡Percentage of DALYs.

§Percentage of all asthma deaths due to occupational exposures.

१Percentage of all asthma DALYs due to occupational exposures.

SDI, socio-demographic index.

asbestosis deaths. The age-standardised death rates were highest in high-income Asia Pacific, East Asia and Oceania (the lowest rates were in Southeast Asia and the Caribbean), and the DALY rates highest in East Asia, Oceania and Southern sub-Saharan Africa (the lowest rates were in the Caribbean and Southeast Asia). Sixty-two percent of the silicosis deaths and 36\% of the CWP deaths occurred in East Asia, and 27\% of the asbestosis deaths occurred in Western Europe, which also had the secondhighest rate (behind East Asia) of silicosis deaths. Western Europe, South Asia and East Asia had the highest number of asbestosis deaths, and East Asia, Australasia and Western Europe had the highest rate of asbestosis deaths (table 4-the rate data for individual pneumoconioses are not shown here).

\section{Changes over time}

For COPD, there was little change ( $4 \%$ rise) in the number of deaths due to occupational exposure to PMGF and SHS between 1990 and 2016, but the (standardised) rate of death from COPD declined by $41 \%$ over this time. For asthmagens, the number of deaths due to occupational exposure increased by $7 \%$ and the rate of death declined by $36 \%$. The number of deaths from pneumoconioses changed minimally (1\%) over this period, but the rate of death from pneumoconioses declined by $41 \%$. Changes in the numbers and rates of DALYs were similar to those seen for deaths, except for asthma, which had a 27\% increase in DALYs between 1990 and 2016. The PAFs for asthma rose considerably over this time (21\% for deaths; $28 \%$ for DALYs), but there was little change in the PAFs for COPD (table 5).

\section{DISCUSSION}

This analysis of the GBD 2016 study has shown there is a considerable burden of chronic respiratory disease worldwide and in all regions arising from exposure to occupational risk factors. Chronic obstructive pulmonary disease is the primary resulting disease, in terms of both deaths and DALYs, but asthma and pneumoconioses are also important. Rates were much higher in males than females for all these disorders, but important in both. The lower female rates reflect the fact that women are less likely to be employed in tasks that involve the relevant exposures. ${ }^{23}$ The results are consistent with those from the overall GBD respiratory analysis. ${ }^{30}$ The decreases in per capita burden for most measures, and the increase for asthma DALYs, result primarily from changes in the relevant PAFs that, in turn, reflect changes in the occupation and industry distribution, which are the basis of the exposure assessments.

\section{PMGF, SHS and COPD}

The global estimate of the PAF for COPD arising from occupational exposure to PMGF and SHS (17\% for DALYs; $16 \%$ for deaths) is consistent with most published findings for individual countries and overall. These have typically reported PAFs of the order 
Table 4 Global occupational-attributable deaths and DALYs from pneumoconioses due to exposure to pneumoconiotic dusts, by region, 2016 (number and rate)*

\begin{tabular}{|c|c|c|c|c|c|c|c|c|c|c|c|c|}
\hline Region & $\begin{array}{l}\text { Deaths } \\
\text { Asb* }^{*}\end{array}$ & $\begin{array}{l}\text { Deaths } \\
\text { CWP** }\end{array}$ & $\begin{array}{l}\text { Deaths } \\
\text { Sil* }^{*}\end{array}$ & $\begin{array}{l}\text { Deaths } \\
\text { Oth* }\end{array}$ & $\begin{array}{l}\text { Deaths } \\
\text { total }\end{array}$ & $\%$ & $\begin{array}{l}\text { Death } \\
\text { ratet }\end{array}$ & YLLs \% ¥ & YLDsł & DALYs & DALYs \% & $\begin{array}{l}\text { DALY } \\
\text { ratef }\end{array}$ \\
\hline High-income North America & 674 & 283 & 118 & 78 & 1153 & 5.4 & 0.7 & 55.6 & 44.4 & 27309 & 4.7 & 16.5 \\
\hline Australasia & 87 & 1 & 11 & 6 & 105 & 0.5 & 0.8 & 88.4 & 11.6 & 1444 & 0.3 & 10.8 \\
\hline High-income Asia Pacific & 345 & 279 & 486 & 477 & 1587 & 7.4 & 1.5 & 95.9 & 4.1 & 21657 & 3.8 & 21.4 \\
\hline Western Europe & 948 & 330 & 1388 & 147 & 2814 & 13.1 & 1.2 & 92.2 & 7.8 & 35385 & 6.1 & 15.5 \\
\hline Southern Latin America & 30 & 17 & 132 & 18 & 197 & 0.9 & 0.7 & 87.9 & 12.1 & 3236 & 0.6 & 12.5 \\
\hline Central Asia & 14 & 3 & 2 & 49 & 68 & 0.3 & 0.3 & 63.9 & 36.1 & 2460 & 0.4 & 8.7 \\
\hline Central Latin America & 38 & 33 & 90 & 75 & 235 & 1.1 & 0.3 & 49.5 & 50.5 & 9294 & 1.6 & 11.4 \\
\hline Andean Latin America & 9 & 7 & 20 & 82 & 117 & 0.5 & 0.7 & 91.3 & 8.7 & 2351 & 0.4 & 12.6 \\
\hline Caribbean & 4 & 2 & 5 & 13 & 24 & 0.1 & 0.1 & 61.9 & 38.1 & 739 & 0.1 & 4.4 \\
\hline Tropical Latin America & 65 & 50 & 235 & 117 & 468 & 2.2 & 0.6 & 85.0 & 15.0 & 12780 & 2.2 & 16.1 \\
\hline South Asia & 547 & 519 & 1089 & 1953 & 4109 & 19.1 & 0.9 & 90.8 & 9.2 & 92603 & 16.0 & 18.4 \\
\hline Southern sub-Saharan Africa & 133 & 12 & 51 & 64 & 260 & 1.2 & 1.3 & 92.6 & 7.4 & 6142 & 1.1 & 28.9 \\
\hline Western sub-Saharan Africa & 10 & 3 & 5 & 38 & 57 & 0.3 & 0.1 & 74.8 & 25.2 & 2099 & 0.4 & 2.4 \\
\hline Eastern sub-Saharan Africa & 47 & 19 & 55 & 108 & 229 & 1.1 & 0.4 & 89.6 & 10.4 & 5745 & 1.0 & 7.5 \\
\hline Central sub-Saharan Africa & 12 & 6 & 15 & 33 & 66 & 0.3 & 0.4 & 90.0 & 10.0 & 1680 & 0.3 & 7.5 \\
\hline High SDI & 2015 & 977 & 1855 & 770 & 5617 & 26.1 & 1.0 & 78.2 & 21.8 & 89690 & 15.5 & 17.3 \\
\hline High-middle SDI & 307 & 324 & 1702 & 569 & 2902 & 13.5 & 0.6 & 59.2 & 40.8 & 90287 & 15.6 & 18.9 \\
\hline Middle SDI & 598 & 778 & 5277 & 1521 & 8173 & 38.0 & 1.0 & 68.6 & 31.4 & 273759 & 47.4 & 31.7 \\
\hline Low-middle SDI & 485 & 548 & 1440 & 1781 & 4253 & 19.8 & 0.8 & 83.4 & 16.6 & 109444 & 19.0 & 18.2 \\
\hline Low SDI & 90 & 57 & 129 & 266 & 542 & 2.5 & 0.4 & 88.6 & 11.4 & 13797 & 2.4 & 8.8 \\
\hline
\end{tabular}

*YLL=years of life lost; YLD=years of life lived with disability; DALYs=disability-adjusted life years; PAF=population attributable fraction; Asb'=asbestosis; CWP=coal workers' pneumoconiosis; 'Sil'=silicosis; 'Oth'=other pneumoconiosis.

tPer 100000 persons.

¥Percentage of DALYs.

SDI, socio-demographic index.

of $10 \%-15 \%$, although much higher values have been estimated, particularly for non-smokers, typically due to differences in the level or type of exposures of the included subjects or the use of different assumptions. ${ }^{1031-35}$ In addition, as smoking rates diminish, the PAF for occupational risk factors will increase. In comparison, the GBD 2016 study estimated PAFs for COPD in regard to smoking and SHS of $43 \%$ and ambient particulate matter pollution of $27 \%{ }^{22}$ The CRA study (covering the year 2000) estimated 318000 deaths and PAFs from occupational exposure of $13 \%$ based on DALYs and 12\% based on deaths. ${ }^{14}$ The Burden of Obstructive Lung Disease (BOLD) study documented a direct relationship between COPD prevalence and number of years worked in dusty jobs. ${ }^{36}$

\section{Asthmagens and asthma}

As with COPD related to occupational exposures, the occupational asthma PAF estimates of 10\% for DALYs and 9\% for deaths from this study are consistent with most published findings for individual countries, which are of the order of 10\%-15\% ${ }^{3781028}$ and comparable to the PAF due to smoking (10\% for DALYs; $14 \%$ for deaths). ${ }^{22}$ The CRA study, which was based on the year 2000, estimated a PAF of $11 \%$ based on DALYs and $17 \%$ based on deaths (and estimated 38000 deaths), ${ }^{14}$ the differences primarily arising from changes in the employment distribution and slight differences in the general methodology. ${ }^{22}$

\section{Pneumoconiotic dusts and pneumoconioses}

Obtaining reliable global information on pneumoconiosis cases is challenging. This analysis identified silicosis as the predominant pneumoconiosis, with much lower numbers of cases of asbestosis and CWP. The increase in rates with age is consistent with the published literature, ${ }^{37}$ and the number of deaths is consistent with the publicly available data for many countries, but also differs considerably for some others for which the estimates here are notably different from the numbers reported in the WHO Mortality Database. ${ }^{38}$ The reason for this is not clear, but presumably is because of the use of different primary data sources and assumptions in the GBD modelling process. It is likely that most of the moderate proportion of pneumoconiosis deaths and DALYs (both 23\%) coded in GBD 2016 as due to 'Other pneumoconioses' were actually due to silicosis, asbestosis or CWP, as these have always been identified as the three main pneumoconioses. The different coding is likely to have arisen due to incomplete coding in the source data and the way this was allocated to specific categories.

\section{Methodological considerations and limitations}

Most of the methodological issues specific to the three main outcomes of interest have already been considered in the relevant sections of the Discussion. The main general uncertainties have been 
Table 5 Change in global occupational-attributable deaths and DALYs from chronic respiratory disease due to occupational exposure to asthmagens, PMGF, SHS and pneumoconiotic dusts between 1990 and 2016, number and per capita (number and percent [95\% uncertainty interval])*

\begin{tabular}{|c|c|c|c|c|c|c|}
\hline \multirow[b]{2}{*}{ Risk factor } & \multicolumn{3}{|l|}{ Deaths } & \multicolumn{3}{|l|}{ DALYs } \\
\hline & $1990 t$ & 2016 & $\%$ change & 1990 & 2016 & $\%$ change \\
\hline \multirow[t]{2}{*}{ Asthmagens } & 35228 & 37574 & 6.7 & 1845494 & 2339480 & 26.8 \\
\hline & (24 103-48462) & $(28362-47936)$ & $(-19.5$ to 36.1$)$ & (1 406629-2327424) & (1 860896-2 923319) & (0.8 to 58.4$)$ \\
\hline \multirow[t]{2}{*}{ PMGF+SHS } & 441702 & 460080 & 4.2 & 9825539 & 10687953 & 8.8 \\
\hline & $(367000-521000)$ & (381500-551300) & (-13.6 to 24.8$)$ & $(8149400-11533600)$ & (9019900-12517000) & (-8.2 to 27.4$)$ \\
\hline \multirow[t]{2}{*}{ Pneumoconiotic dusts } & 21209 & 21488 & 1.3 & 567941 & 576977 & 1.6 \\
\hline & $(16000-31400)$ & $(17900-25400)$ & $(-15.6$ to 19.8$)$ & $(442576-832555)$ & (493632-673528) & $(-13.1$ to 18.6$)$ \\
\hline \multirow[t]{2}{*}{ Total } & 498139 & 519142 & 4.2 & 12238974 & 13604410 & 11.2 \\
\hline & $(407000-600900)$ & $(427800-624600)$ & $(-14.9$ to 25.4$)$ & (10667 700-13 881 800) & $(11912000-15502,800)$ & $(-2.7$ to 26.7$)$ \\
\hline & \multicolumn{3}{|c|}{ Deaths per 100000 persons } & \multicolumn{3}{|c|}{ DALYs per 100000 persons } \\
\hline Risk factor & $1990 t$ & 2016 & $\%$ change & 1990 & 2016 & $\%$ change \\
\hline \multirow[t]{2}{*}{ Asthmagens } & 2.2 & 1.4 & -36.0 & 108 & 85 & -21.4 \\
\hline & $(1.5-3.0)$ & $(1.0-1.8)$ & $(-51.7$ to -18.1$)$ & $(82-136)$ & $(67-106)$ & $(-37.5$ to -1.7$)$ \\
\hline \multirow[t]{2}{*}{$\mathrm{PMGF}+\mathrm{SHS}$} & 31.0 & 18.2 & -41.3 & 653 & 410 & -37.1 \\
\hline & $(26.8-36.5)$ & $(15.0-21.9)$ & $(-51.7$ to -29.4$)$ & $(545-768)$ & $(346-482)$ & $(-47.1$ to -26.1$)$ \\
\hline \multirow[t]{2}{*}{ Pneumoconiotic dusts } & 1.43 & 0.84 & -41.3 & 36 & 22 & -39.7 \\
\hline & $(1.08-2.10)$ & $(0.70-0.99)$ & $(-51.0$ to -30.8$)$ & $(28-53)$ & $(19-26)$ & $(-48.5$ to -29.7$)$ \\
\hline \multirow[t]{2}{*}{ Total } & 34.6 & 20.4 & -40.9 & 796 & 517 & -35.1 \\
\hline & $(28.4-41.6)$ & $(16.7-24.6)$ & $(-51.6$ to -28.8$)$ & $(655-957)$ & $(431-613)$ & $(-45.8$ to -23.0$)$ \\
\hline & \multicolumn{3}{|c|}{ Population attributable fraction (deaths) (\%) } & \multicolumn{3}{|c|}{ Population attributable fraction (DALYs) (\%) } \\
\hline Risk factor & $1990 *$ & 2016 & $\%$ change & 1990 & 2016 & $\%$ change \\
\hline Asthmagens & $7.4(6.1-8.8)$ & $8.9(7.8-10.1)$ & $20.6(5.4-36.0)$ & $7.7(6.8-8.8)$ & $9.9(9.0-10.9)$ & $27.6(16.0-40.3)$ \\
\hline \multirow[t]{2}{*}{$\mathrm{PMGF}+\mathrm{SHS}$} & 16.3 & 15.7 & -3.6 & 16.4 & 16.9 & 2.5 \\
\hline & $(13.7-19.1)$ & $(13.8-18.6)$ & $(-20.0$ to 14.0$)$ & $(14.0-19.0)$ & $(14.3-19.7)$ & (-13.0 to 19.8$)$ \\
\hline
\end{tabular}

${ }^{*}$ DALYs=disability-adjusted Life Years; PMGF=particulate matter, gases and fumes; SHS=secondhand smoke.

tThe numbers in brackets in the whole table are $95 \%$ uncertainty intervals.

considered in detail in the companion overview paper. $^{21}$ Issues of particular relevance to the presented analysis included basing exposure prevalence estimates on industry (for PMGF and SHS) and occupation (for asthmagens); uncertainty in the prevalence and level of exposure to PMGF overall and in different industries; the potential for mismatch between the relative risk estimates used and the exposure circumstances to which they have been applied; not explicitly taking into account the potential effect of differences in smoking habits and environmental exposures between regions and over time; probable heterogeneity in terms of how chronic respiratory conditions are identified, diagnosed and managed worldwide; and not including some potentially relevant risk factors and outcomes such as respiratory infections, ${ }^{39}$ other occupational causes of fibrosis apart from pneumoconioses and lung disease arising from nanoparticle exposure. ${ }^{40}$ For both COPD and asthma, the extent and effect of any mismatch between the exposure and the relative risk estimate applied in LMI countries are not clear. It would be helpful to have usable information on this from LMI countries, which might allow different risk estimates to be applied in these countries if appropriate. However, currently the necessary data are not available.

\section{Implications and uses of the data}

The main finding of this study is that workplace exposures resulting in COPD, asthma and pneumoconioses remain important contributors to the burden of disease in all regions of the world. The relevant exposures are respiratory and it should be possible to minimise all (or most), and in some instances to essentially eliminate them, through appropriate commitment to, and implementation of, exposure control interventions to decrease the airborne exposure levels of the relevant hazards. However, it must be recognised that there are a range of PMGF implicated as increasing the risk of COPD and hundreds of known occupational asthmagens. Elimination or appropriate control of many of these exposures will take considerable resources and effort and requires continued vigilance. The study does not provide information on the cost or practicality of eliminating or better controlling the relevant exposures, and the results for COPD and pneumoconioses largely reflect past exposures. However, the high burden of COPD cases suggests the relevant exposures should be a priority in the area of occupational airborne exposures resulting in chronic respiratory disease. The findings also have implications for healthcare costs and social protection in older individuals. Finally, further investment in country-level data sources, especially in LMI countries, would help improve the accuracy and usefulness of the estimates generated by the GBD study.

\section{Conclusions}

There are many respiratory conditions that can arise directly, or indirectly, from work. The results from this study indicate that non-malignant/non-infectious respiratory diseases arising from occupational exposures are an important cause of death and disability worldwide. Many of these cases should be preventable by adopting better health and safety approaches, particularly through improved engineering and working conditions. 
Collaborators Collaborators details are as follows: Tim Driscoll, Kyle Steenland, Neil Pearce, Lesley Rushton, Sally J. Hutchings, Kurt Straif, Degu Abate, Dilaram Acharya, Anurag Agrawal, Fares Alahdab, Kefyalew Addis Alene, Sofia Androudi, Mina Anjomshoa, Carl Abelardo T. Antonio, Olatunde Aremu, Zerihun Ataro, Alaa Badawi, Joseph Adel Mattar Banoub, Suzanne Lyn Barker-Collo, Neeraj Bedi, Derrick A. Bennett, Robert Bernstein214, Mircea Beuran, Krittika Bhattacharyya, Ali Bijani, Zahid A. Butt, Juan J. Carrero, Carlos A. Castañeda-Orjuela, Odgerel Chimed-Ochir, Lalit Dandona, Rakhi Dandona, Anh Kim Dang, Ahmad Daryani, Beruk Berhanu Desalegn, Samath Dhamminda Dharmaratne, Shirin Djalalinia, Eleonora Dubljanin, Soheil Ebrahimpour, Ziad El-Khatib, Mohammad Fareed, Andre Faro, Eduarda Fernandes, Florian Fischer, Takeshi Fukumoto, Silvano Gallus, Teklu Gebrehiwo Gebremichael, Kebede Embaye Gezae, Ayman Grada, Yuming Guo, Rahul Gupta, Arvin Haj-Mirzaian, Arya Haj-Mirzaian, Samer Hamidi, Mehedi Hasan, Milad Hasankhani, Simon I. Hay, Chi Linh Hoang, Michael K. Hole, H Dean Hosgood, Mihaela Hostiuc, Sorin Hostiuc, Seyed Sina Naghibi Irvani, Sheikh Mohammed Shariful Islam, Mihajlo Jakovljevic, Ravi Prakash Jha, Jost B. Jonas, Amaha Kahsay, Amir Kasaeian, Norito Kawakami, Yousef Saleh Khader, Morteza Abdullatif Khafaie, Ejaz Ahmad Khan, Mohammad Hossein Khosravi, Jagdish Khubchandani, Yun Jin Kim, Ruth W. Kimokoti, Adnan Kisa, Manolis Kogevinas215, Soewarta Kosen, Parvaiz A. Koul, Ai Koyanagi, Barthelemy Kuate Defo, G Anil Kumar, Dharmesh Kumar Lal, Arman Latifi, James Leigh, Miriam Levi, Shanshan Li, Shai Linn, Narayan Bahadur Mahotra, Marek Majdan, Reza Malekzadeh, Mohammad Ali Mansournia, Francisco Rogerlândio Martins-Melo, Benjamin Ballard Massenburg, Varshil Mehta, Addisu Melese, Mulugeta Melku, Ziad A. Memish, Walter Mendoza, Tuomo J. Meretoja, Tomislav Mestrovic, GK Mini, Erkin M. Mirrakhimov, Babak Moazen, Naser Mohammad Gholi Mezerji, Shafiu Mohammed, Ali H Mokdad, Lorenzo Monasta, Yoshan Moodley, Mahmood Moosazadeh, Ghobad Moradi, Lidia Morawska, Shane Douglas Morrison, Seyyed Meysam Mousavi, Ghulam Mustafa, Vinay Nangia, lonut Negoi, Ruxandra Irina Negoi, Cuong Tat Nguyen, Trang Huyen Nguyen, Molly R. Nixon, Richard Ofori-Asenso, Felix Akpojene Ogbo, Andrew T. Olagunju, Bolajoko Olubukunola Olusanya, Mahesh PA, Songhomitra Panda-Jonas, Eun-Kee Park, Sanghamitra Pati, Mostafa Qorbani, Anwar Rafay, Alireza Rafiei, Fakher Rahim, Vafa Rahimi-Movaghar, Fatemeh Rajati, Robert C. Reiner, Satar Rezaei, Leonardo Roever, Luca Ronfani, Gholamreza Roshandel, Basema Saddik, Saeid Safiri, Mohammad Ali Sahraian, Abdallah M. Samy, David C. Schwebel, Sadaf G. Sepanlou, Berrin Serdar, Masood Ali Shaikh, Aziz Sheikh, Mika Shigematsu, Rahman Shiri, Reza Shirkoohi, Si Si, João Pedro Silva, Dhirendra Narain Sinha, Moslem Soofi, Joan B. Soriano, Chandrashekhar T. Sreeramareddy, Jeffrey D. Stanaway, Mark A. Stokes, Mu'awiyyah Babale Sufiyan, Ipsita Sutradhar, Rafael Tabarés-Seisdedos, Ken Takahashi, Yonatal Mesfin Tefera, Mohamad-Hani Temsah, Marcos Roberto Tovani-Palone, Bach Xuan Tran, Khanh Bao Tran, Lorainne Tudor Car, Irfan Ullah, Pascual R. Valdez, Job F. M. van Boven, Tommi Juhani Vasankari, Francesco S. Violante, Giang Thu Vu, Gregory R. Wagner, Yasir Waheed, Yuan-Pang Wang, Biruck Desalegn Yirsaw, Naohiro Yonemoto, Chuanhua Yu, Mohammad Zamani, and Stephen S. Lim (online supplementary file)

Contributors The draft manuscript was prepared by Tim Driscoll, with input from Sally Hutchings, Lesley Rushton, Kyle Steenland and Kurt Straif. All listed authors have contributed appropriately to the GBD project and to the review and modification of the manuscript. The final manuscript was prepared by TD following comments from co-authors and Journal reviewers and editors.

Funding The overall GBD study is partly funded by the Bill \& Melinda Gates Foundation. The work reported in this paper was partly supported by funding from the World Health Organization. The funders had no role in the study design, data collection, data analysis, data interpretation or writing of the report. The authors had access to the data in the study and the final responsibility to submit the paper.

Disclaimer The views expressed are those of the authors and not necessarily those of the NHS, the NIHR or the Department of Health.

Competing interests CATA reports personal fees from Johnson \& Johnson (Philippines), Inc., outside the submitted work. NK reports personal fees from Junpukai Foundation and Softbank, Co.; and grants from Fujitsu, LTD, Fujitsu Software Technologies, LTD and Softbank, Co., outside the submitted work. JK reports grants from Merck Pharmaceuticals, outside the submitted work. TJM reports grants from Cancer Foundation Finland sr., during the conduct of the study.

\section{Patient consent for publication Not required.}

Provenance and peer review Not commissioned; externally peer reviewed.

Data availability statement Data are available in a public, open access repository. The data on which this analysis is based are available on the GBD Compare web site (https:// vizhub. healthdata. org/ gbd- compare/). Some of the raw data (where data owners give permission or where it is already public access) is available on the data section of the IHME GBD web site (http://www. healthdata. org/gbd/ data).

Open access This is an open access article distributed in accordance with the Creative Commons Attribution 4.0 Unported (CC BY 4.0) license, which permits others to copy, redistribute, remix, transform and build upon this work for any purpose, provided the original work is properly cited, a link to the licence is given, and indication of whether changes were made. See: https://creativecommons.org/ licenses/by/4.0/.

\section{REFERENCES}

1 Fishwick D, Sen D, Barber C, et al. Occupational chronic obstructive pulmonary disease: a standard of care. Occup Med 2015;65:270-82.

2 Omland Øyvind, Würtz ET, Aasen TB, et al. Occupational chronic obstructive pulmonary disease: a systematic literature review. Scand J Work Environ Health 2014;40:19-35.

3 Tarlo S, Malo J. Fourth jack Pepys workshop on asthma in the workplace participants. An official American thoracic Society proceedings: work-related asthma and airway diseases. presentations and discussion from the fourth jack Pepys workshop on asthma in the workplace. Ann Am Thorac Soc 2013;10:S17-S2.

4 Blanc PD. Occupation and COPD: a brief review. J Asthma 2012;49:2-4.

5 Blanc PD, Iribarren C, Trupin L, et al. Occupational exposures and the risk of COPD: dusty trades revisited. Thorax 2009;64:6-12

6 Rushton L. Occupational causes of chronic obstructive pulmonary disease. Rev Environ Health 2007;22:195-212.

7 Karjalainen A, Kurppa K, Martikainen R, et al. Exploration of asthma risk by occupation - extended analysis of an incidence study of the Finnish population. Scand J Work Environ Health 2002;28:49-57.

8 Karjalainen A, Kurppa K, Martikanen R, et al. Work is related to a substantial portion of adult-onset asthma incidence in the Finnish population. Am J Respir Crit Care Med 2001;164:565-8.

9 Kogevinas M, Zock J-P, Jarvis D, et al. Exposure to substances in the workplace and new-onset asthma: an international prospective population-based study (ECRHS-II). The Lancet 2007;370:336-41.

10 Eisner MD, Anthonisen N, Coultas D, et al. An official American thoracic Society public policy statement: novel risk factors and the global burden of chronic obstructive pulmonary disease. Am J Respir Crit Care Med 2010:182:693-718.

11 Blackley DJ, Halldin CN, Laney AS. Continued Increase in Prevalence of Coal Workers' Pneumoconiosis in the United States, 1970-2017. Am J Public Health 2018;108:1220-2

12 Hoy RF, Baird T, Hammerschlag G, et al. Artificial stone-associated silicosis: a rapidly emerging occupational lung disease. Occup Environ Med 2018;75:3-5.

13 Nelson DI, Concha-Barrientos M, Driscoll T, et al. The global burden of selected occupational diseases and injury risks: methodology and summary. Am J Ind Med 2005;48:400-18

14 Driscoll T, Nelson DI, Steenland K, et al. The global burden of non-malignant respiratory disease due to occupational airborne exposures. Am J Ind Med 2005:48:432-45.

15 Lim SS, Vos T, Flaxman AD, et al. A comparative risk assessment of burden of disease and injury attributable to 67 risk factors and risk factor clusters in 21 regions, 1990-2010: a systematic analysis for the global burden of disease study 2010. The Lancet 2012;380:2224-60.

16 GBD. Global, regional, and national comparative risk assessment of 79 behavioural, environmental and occupational, and metabolic risks or clusters of risks in 188 countries, 1990-2013: a systematic analysis for the global burden of disease study 2013. Lancet 2015;388:1659-724

17 Forouzanfar MH, Afshin A, Alexander LT, et al. Global, regional, and national comparative risk assessment of 79 behavioural, environmental and occupational, and metabolic risks or clusters of risks, 1990-2015: a systematic analysis for the global burden of disease study 2015. Lancet 2016;388:1659-724.

18 Gakidou E, Afshin A, Abajobir AA, et al. Global, regional, and national comparative risk assessment of 84 behavioural, environmental and occupational, and metabolic risks or clusters of risks, 1990-2016: a systematic analysis for the global burden of disease study 2016. Lancet 2017;390:1345-422.

19 Stanaway JD, Afshin A, Gakidou E, et al. Global, regional, and national comparative risk assessment of 84 behavioural, environmental and occupational, and metabolic risks or clusters of risks for 195 countries and territories, 1990-2017: a systematic analysis for the global burden of disease study 2017. Lancet 2018;392:1923-94.

20 GBD 2016 Occupational Carcinogens Collaborators. Global and regional burden of cancer in 2016 arising from occupational exposure to selected carcinogens: a systematic analysis for the global burden of disease study 2016. Occup Environ Med 2020:77:151-9.

21 GBD 2016 Occupational Risk Factors Collaborators. Global and regional burden of disease and injury in 2016 arising from occupational exposures: a systematic analysis for the global burden of disease study 2016. Occup Environ Med 2020;77:133-41.

22 Institute for Health Metrics and Evaluation. GBD compare. Seattle, WA: IHME, University of Washington, 2017.

23 International Labour Office. ILOSTAT database. Geneva: ILO, 2015. 
24 Levin M. The occurrence of lung cancer in man. Acta Unio Internationalis Contra Cancrum 1959;9:531-41.

25 Weinmann S, Vollmer WM, Breen V, et al. Copd and occupational exposures: a casecontrol study. J Occup Environ Med 2008;50:561-9.

26 Vestbo J, Hurd SS, Agustí AG, et al. Global strategy for the diagnosis, management, and prevention of chronic obstructive pulmonary disease. Am J Respir Crit Care Med 2013; 187:347-65

27 Kauppinen T, Toikkanen J, Pedersen D, et al. Occupational exposure to carcinogens in the European Union. Occup Environ Med 2000;57:10-18.

28 Kogevinas M, Anto J, Sunyer J, et al. Occupational asthma in Europe. Lancet 1999:353:1750-4.

29 Wang $\mathrm{H}, \mathrm{Abajobir} \mathrm{AA}$, Abate $\mathrm{KH}$, et al. Global, regional, and national under-5 mortality, adult mortality, age-specific mortality, and life expectancy, 1970-2016: a systematic analysis for the global burden of disease study 2016. The Lancet 2017:390:1084-150.

30 Soriano JB, Abajobir AA, Abate KH, et al. Global, regional, and national deaths, prevalence, disability-adjusted life years, and years lived with disability for chronic obstructive pulmonary disease and asthma, 1990-2015: a systematic analysis for the global burden of disease study 2015. Lancet Respir Med 2017:5:691-706.

31 Balmes J, Becklake M, Blanc P, et al. American thoracic Society statement: occupational contribution to the burden of airway disease. Am J Respir Crit Care Med 2003;167:787-97.
32 Meldrum Met al. The role of occupation in the development of chronic obstructive pulmonary disease (COPD). Occup Environ Med 2005;62:212-4.

33 Mehta AJ, Miedinger D, Keidel D, et al. Occupational exposure to dusts, gases, and fumes and incidence of chronic obstructive pulmonary disease in the Swiss cohort study on air pollution and lung and heart diseases in adults. Am J Respir Crit Care Med 2012;185:1292-300.

34 Lytras T, Kogevinas M, Kromhout H, et al. Occupational exposures and 20-year incidence of COPD: the European community respiratory health survey. Thorax 2018;73:1008-15.

35 Würtz ET, Schlünssen V, Malling TH, et al. Occupational COPD among Danish neversmokers: a population-based study. Occup Environ Med 2015;72:456-9.

36 Hooper R, Burney P, Vollmer WM, et al. Risk factors for COPD spirometrically defined from the lower limit of normal in the BOLD project. Eur Respir $J$ 2012;39:1343-53

37 Darnton A, Hodgson J, Benson P, et al. Mortality from asbestosis and mesothelioma in Britain by birth cohort. Occup Med 2012;62:549-52.

38 World Health Organization. Who mortality database. Geneva: WHO, 2016

39 Baussano I, Nunn P, Williams B, et al. Tuberculosis among health care workers. Emerg Infect Dis 2011;17:488-94.

40 Traboulsi H, Guerrina N, lu M, et al. Inhaled pollutants: the molecular scene behind respiratory and systemic diseases associated with ultrafine particulate matter. Int J Mol Sci 2017;18:243 